\title{
El bienestar animal en sistemas productivos de ovinos-caprinos en Colombia
}

\author{
Víctor Manuel Acero-Plazas*, $\mathrm{MV}_{1}$
}

${ }_{1}$ Asociación Nacional de Médicos Veterinarios de Colombia (amevec), Bogotá, Colombia

Recibido: 10 de noviembre de 2014. Aprobado: 15 de diciembre de 2014.

*Autor de correspondencia: Víctor Manuel Acero-Plazas. Asociación Nacional de Médicos Veterinarios de Colombia, calle 101 \# 71A - 52, Barrio Pontevedra, Bogotá, Colombia. Teléfono: (57) 1 6434135. Correo electrónico: sepulvic@gmail.com

Cómo citar este artículo: Acero-Plazas VM. El bienestar animal en sistemas productivos de ovinos-caprinos en Colombia. Spei Domus. 2014;10(20):57-62. doi: http:// dx.doi.org/10.16925/sp.v10i21.918

Resumen. En Colombia, los sistemas pecuarios o sistemas productivos están enfocados hacia las especies con mayor "potencial" de exportación o de mayor consumo nacional, las cuales son: aves (carne, huevos, genética), bovinos (leche, carne, genética), porcinos (carne, genética) y peces. En menor escala, están los búfalos, caprinos-ovinos, conejos y cuyes. De hecho, la normatividad nacional en cuanto a la producción primaria ha estado enfocada en las buenas prácticas ganaderas, avícolas, porcícolas, piscícolas, y actualmente existen normas para el sacrificio de bovinos, porcinos y aves. La industria de estas especies se encuentra bien desarrollada en general, aunque con algunos vacíos en bienestar animal, trazabilidad, sistemas de vigilancia y control de enfermedades, sistemas de inocuidad y cumplimiento de normatividad internacional para la exportación. Datos del 2012 muestran que la distribución de la producción pecuaria de Colombia está constituida principalmente por 27 millones de cabezas de bovinos, 25 millones de aves (pollos/gallinas), 2,5 millones de equinos, 1,6 millones de porcinos, 1,3 millones de caprinos, 1,1 millones de ovinos, entre otros. Esto demuestra el desbalance del sector agropecuario, donde hay una clara tendencia hacia las especies productivas tradicionales. Entre ovejas y cabras pueden sumar hoy en día unos 2,5 millones de cabezas.

Palabras clave: bienestar, ovinos-caprinos, sistemas productivos.

\section{Animal Welfare in Colombian Sheep-Goat Production Systems}

Abstract. In Colombia, livestock systems or production systems are focused on species with the greatest export potential or highest national consumption. These are poultry (meat, eggs, genetics), cattle (milk, meat, genetics), pigs (meat, genetics) and fish. There is also smallerscale production of buffalos, sheep/goats, rabbits and guinea pigs. National standards related to primary production have centered on good practices for cattle, poultry and pigs, and there are currently norms for the slaughter of these animals. The industry for these species is generally well developed, albeit with some gaps in the areas of animal welfare, traceability, and disease control and surveillance systems, food-safety systems and compliance with international standards for exportation. Data from 2012 shows that Colombian livestock production primarily consists of 27 million head of cattle, 25 million birds (chickens/hens), 2.5 million horses, 1.6 million pigs, 1.3 million goats, and 1.1 million sheep, among other animals. This shows an imbalance in the agricultural sector, where there is a clear tendency towards traditional production species. Today, there are approximately 2.5 million head of sheep and goats when the two species are taken together.

Keywords: welfare, sheep-goats, production systems.

\section{O bem-estar em sistemas produtivos de ovino-caprinos na Colômbia}

Resumo. Na Colômbia, os sistemas pecuários ou sistemas produtivos estão focados nas espécies com maior "potencial" de exportação ou de maior consumo nacional, as quais são: aves (carne, ovos, genética), bovinos (leite, carne, genética), suínos (carne, genética) e peixes. Em menor escala estão os búfalos, caprino-ovinos, coelhos e cuyes. $\mathrm{Na}$ verdade, a regulamentação nacional em matéria de produção primária tem sido focada nas boas práticas pecuárias, avícolas, suínas, piscicultura; e atualmente existem normas para o sacrifício de bovinos, suínos e aves. A indústria destas espécies é bem desenvolvida, em geral, apesar de algumas lacunas no bem-estar animal, rastreabilidade, sistemas de vigilância e controle de doenças, sistemas de segurança e cumprimento das normas internacionais para exportação. Dados de 2012 mostram que a distribuição da produção pecuária da Colômbia está constituída principalmente por 27 milhões de cabeças de gado, 25 milhões de aves (frangos/galinhas), 2,5 milhões de equinos, 1,6 milhões de suínos, 1,3 milhões de caprinos, 1,1 milhões de ovinos; entre outros. Isso demonstra o desequilíbrio do setor agropecuário, em que há uma clara tendência para as espécies produtivas tradicionais. Entre ovelhas e cabras podem somar hoje em dia uns 2,5 milhões de cabeças.

Palavras-chave: bem-estar, ovino-caprinos, sistemas produtivos. 


\section{Contexto de la cadena ovino-caprina en Colombia ${ }^{1}$}

La producción ovino-caprina en Colombia está caracterizada por razas introducidas, razas criollas y cruzamientos alternos con el fin de fortalecer el vigor híbrido, para carne, líneas maternas o producción de leche de cabra para elaboración de subproductos. Estos sistemas productivos son extensivos y semi-intensivos, con tecnología de baja a media.

La cadena ovino-caprina en Colombia está caracterizada por dos eslabones: producción de cárnicos y productos artesanales, y la cadena de producción de leche y derivados; aunque se pueden encontrar explotaciones con los dos sistemas. Para el caso de la carne, se crían reproductores y vientres para mantener la genética y abastecer las granjas que necesitan machos o hembras. De igual manera, una parte de los animales es destinada a la ceba y al posterior sacrificio. De este proceso, se obtienen las canales y subproductos tales como pieles, vísceras y contenido ruminal, productos que son utilizados en procesos industriales.

Para el caso de la cadena láctea, existen apriscos dedicados a la selección y cruzamientos de ejemplares para leche, conformando pies de cría. La leche es transportada y pasteurizada en sólo aquellos casos donde hay productores organizados o empresas que tienen tanque frío y agroindustria de la leche de cabra, dándole valor agregado al producto [1].

En ambos eslabones, la comercialización de todos estos productos juega un papel muy importante. Sin embargo, la agroindustria es poco desarrollada, con un censo escaso de pequeñas empresas dedicadas a la explotación de ovejas o cabras, que procesa productos cárnicos o lácteos. De las empresas dedicadas al proceso de productos cárnicos, la mayoría se encuentran lejos de las pocas plantas autorizadas, las demás reciben las canales. En Colombia, la producción de cabras y ovejas está en manos de pequeños productores, que tienen poco acceso a la tecnología, aunque estas explotaciones cumplen una función económica importante en estas comunidades, siendo el eje central de la agricultura familiar a baja escala, establecida en zonas de poblaciones desatendidas o de bajos recursos.

Este artículo de reflexión es producto de la participación del autor en el curso virtual sobre Bienestar en Animales de Producción realizado por la Protección Animal Mundial (WPA, por sus siglas en inglés) en el 2014.
En el 2011, Colombia contaba con cerca de 1,5 millones de cabezas de ovinos, aunque el consumo per cápita de este producto es ampliamente superado por el consumo de los demás productos (pollo, bovino y porcino), debido al escaso desarrollo de su cadena productiva y que son muy pocos los establecimientos autorizados para el sacrificio de esta especie [2].

El precio por kilo de carne que se le paga al productor puede oscilar entre 2 y 3 dólares, mientras que en los supermercados o restaurantes el costo puede oscilar entre 9 y 10 dólares. Las razas de ovinos que más se comercializan en el país son: Camuro (ovino de pelo colombiano para carne), Katahdin, Pelibuey, Dorset, Suffolk, Black Faced, Texel, Hampshire, y Romney. El precio del kilo en pie puede oscilar entre 2 y 2,5 dólares, dependiendo de la calidad y la zona. En cuanto a las razas caprinas, se manejan: Alpina, Nubiana, Saanen, Boer, Toggenburg, entre otras, y la raza guajira o criolla, resultado del cruce de cabras nativas y otras razas.

\section{Métodos de manejo y crianza}

El manejo de los ovinos se realiza de manera extensiva en su mayoría, salvo algunas excepciones de sistemas semi-intensivos, con alimentación compuesta por forrajes nativos, en algunos casos forrajes mejorados, suplementos y concentrados en otros casos. Las instalaciones están comprendidas por corrales de cría, reproducción, alimentación y manejo, de manera tradicional. La falta de asistencia técnica y la aplicación de tecnología en este tipo de explotaciones se deben a que pertenecen a familias campesinas y pequeños productores, por lo que no tienen acceso a estas herramientas. Algunos factores que componen los sistemas extensivos de pequeños rumiantes se pueden observar en la tabla 1. Incluso, esos sistemas de producción ovina y caprina constituyen el medio de subsistencia de familias campesinas en diferentes zonas del país, aún con baja tecnología y bajos indicadores zootécnicos productivos [3].

Para el caso de aquellos sistemas semi-intensivos o intensivos, algunos de los factores que los componen los podemos observar en la tabla 2 [4].

Los componentes que integran estos dos sistemas denotan claramente que no tienen en cuenta el bienestar animal, o si lo tienen en cuenta es de manera muy pobre. El sistema donde posiblemente se vea más afectado el bienestar animal es el extensivo. 
Tabla 1. Componentes de los sistemas extensivos de pequeños rumiantes en Colombia

\begin{tabular}{|l|l|}
\hline \multicolumn{1}{|c|}{ Factor } & \multicolumn{1}{c|}{ Observaciones } \\
\hline $\begin{array}{l}\text { Aspectos } \\
\text { socioeconómicos del } \\
\text { productor }\end{array}$ & $\begin{array}{l}\text { Objetivo de producción (ahorro, autoconsumo) } \\
\text { No asistencia técnica } \\
\text { No acceso a tecnología }\end{array}$ \\
\hline Genética & $\begin{array}{l}\text { Animales criollos, rústicos o poco } \\
\text { productivos }\end{array}$ \\
\hline Nutrición & Pobre condición corporal \\
\hline Manejo & $\begin{array}{l}\text { Instalaciones rústicas } \\
\text { Pobre higiene } \\
\text { Encierro nocturno }\end{array}$ \\
\hline Alimentación & Depende del recurso forrajero disponible \\
\hline Sanidad & $\begin{array}{l}\text { Problemas relacionados con el ambiente } \\
\text { No hay medicina preventiva }\end{array}$ \\
\hline Reproducción & $\begin{array}{l}\text { Sin control } \\
\text { Monta continua } \\
\text { Consanguinidad }\end{array}$ \\
\hline
\end{tabular}

Fuente: [4]

Tabla 2. Componentes de los sistemas intensivos de pequeños rumiantes en Colombia

\begin{tabular}{|l|l|}
\hline \multicolumn{1}{|c|}{ Factor } & \multicolumn{1}{c|}{ Observaciones } \\
\hline $\begin{array}{l}\text { Aspectos } \\
\text { socioeconómicos del } \\
\text { productor }\end{array}$ & $\begin{array}{l}\text { Objetivo de producción (negocio) } \\
\text { Alto poder adquisitivo } \\
\text { Disponibilidad asesoría técnica }\end{array}$ \\
\hline Genética & Razas especializadas \\
\hline Nutrición & Buena condición corporal \\
\hline Manejo & $\begin{array}{l}\text { Instalaciones adecuadas } \\
\text { Buena higiene }\end{array}$ \\
\hline Alimentación & $\begin{array}{l}\text { Controlada, concentrados y forrajes de buena } \\
\text { calidad }\end{array}$ \\
\hline Sanidad & $\begin{array}{l}\text { Problemas relacionados con la producción } \\
\text { (estrés, hacinamiento) } \\
\text { Programas preventivos }\end{array}$ \\
\hline Reproducción & $\begin{array}{l}\text { Controlada } \\
\text { Programa de montas por época }\end{array}$ \\
\hline
\end{tabular}

Fuente: [4]

Para el caso de las ovejas, los principales indicadores se pueden resumir en la tabla 3.

Para el caso de las cabras, en sistemas productivos tropicales, en la tabla 4 podemos observar algunos parámetros productivos.

\section{Engorde y sacrificio}

Según la Asociación de Criadores de Ganado Ovino de Colombia (Asoovinos), el sacrificio formal de ovinos es bajo, debido principalmente a la ausencia de plantas de beneficio autorizadas para estas especies, por lo que un gran número de animales son sacrificados de manera informal en fincas y plazas de mercado. Según el Boletín de Sacrificio de Ganado en Colombia, emitido por el DANE, en el 2009 fueron sacrificados formalmente en el
Tabla 3. Parámetros productivos de ovinos en Colombia

\begin{tabular}{|l|c|c|}
\hline \multicolumn{1}{|c|}{ Parámetro } & $\begin{array}{c}\text { Tecnología } \\
\text { baja }\end{array}$ & $\begin{array}{c}\text { Tecnología } \\
\text { media }\end{array}$ \\
\hline Peso al nacer (kg) & 1,2 & 2,5 \\
\hline Edad al destete (días) & 60 & 60 \\
\hline Peso al destete (kg) & 14 & 15 \\
\hline Ganancia de peso diaria (g) & 50 a 100 & 80 a 160 \\
\hline Peso al primer servicio (kg) & Menor a 35 & 35 \\
\hline Partos (hembra/año) & 1 & 1,3 \\
\hline Edad al sacrificio (días) & 300 & 240 \\
\hline Peso al sacrificio (kg) & Menor a 30 & 30 \\
\hline Rendimiento en canal (\%) & Menor a 50 & 50 \\
\hline
\end{tabular}

Fuente: elaboración propia

Tabla 4. Parámetros productivos de cabras en el trópico

\begin{tabular}{|l|c|c|c|}
\hline \multicolumn{1}{|c|}{ Parámetro } & Razas europeas & Razas nativas & Cruces (f1) \\
\hline $\begin{array}{l}\text { Peso al nacer } \\
(\mathrm{kg})\end{array}$ & 2,8 & 1,5 & 3,4 \\
\hline $\begin{array}{l}\text { Peso a los } 3 \\
\text { meses (kg) }\end{array}$ & 11,4 & 8,1 & 12,4 \\
\hline
\end{tabular}

Fuente: [5]

país un total de 13094 animales, con un peso en pie total de $508704 \mathrm{~kg}$ y un peso en canal total de $251715 \mathrm{~kg}$, en una relación de 6718 machos contra 6376 hembras. Para el 2010, se sacrificaron 17105 animales con un peso en pie total de $620782 \mathrm{~kg}$ y un peso en canal total de 308 $624 \mathrm{~kg}$, en una relación de 9224 machos contra 7882 hembras [6]. Según estos datos, podemos deducir que el peso promedio al sacrificio es de 38,8 kilos, lo cual es bajo si se tienen en cuenta otros indicadores de producción de otras regiones similares. El consumo de carne ovina y caprina se ha mantenido estable en los últimos años, para el 2010 los datos indican que fue de 0,162 y $0,160 \mathrm{~kg} /$ persona al año, respectivamente. Otros datos indican que en el 2005, en Colombia hubo un consumo per cápita de 310 gramos [1].

\section{Bienestar animal en la especie ovino- caprina en Colombia e indicadores}

En los ovinos y caprinos, en Colombia, no existe normatividad vigente ni de carácter obligatorio para aplicar o poner en práctica el bienestar en estas especies. Incluso, aún no está normado de manera obligatoria en las especies (aves, bovinos, porcinos), sólo se da de manera opcional o como valor agregado, cuando debería ser de cumplimiento obligatorio. En la tabla 5, observamos los principales métodos para medir el bienestar animal en caprinos-ovinos, y su posible relación con enfermedades [7]. 
Tabla 5. Diferentes métodos para determinar el bienestar animal y su relación con alguna enfermedad

\begin{tabular}{|l|l|l|l|}
\hline \multicolumn{1}{|c|}{ Método } & \multicolumn{1}{|c|}{ Facilidad de uso } & \multicolumn{1}{c|}{ Aplicación } & \multicolumn{1}{c|}{ Inconvenientes } \\
\hline Libertades & Se utiliza en varios países & $\begin{array}{l}\text { Puede ser utilizada en granjas y } \\
\text { establecimientos comerciales }\end{array}$ & $\begin{array}{l}\text { Puede ser subjetiva según la } \\
\text { valoración de los observadores }\end{array}$ \\
\hline $\begin{array}{l}\text { Algoritmos (escalas análogas } \\
\text { visuales) }\end{array}$ & $\begin{array}{l}\text { Utilizado en medicina para evaluar } \\
\text { escalas, por ejemplo, el dolor }\end{array}$ & $\begin{array}{l}\text { Util para propietarios o cuidadores } \\
\text { de animales, donde hay una } \\
\text { observación diaria }\end{array}$ & $\begin{array}{l}\text { Puede ser subjetiva según la } \\
\text { valoración de los observadores }\end{array}$ \\
\hline Signos clínicos & $\begin{array}{l}\text { Puede haber técnicas invasivas y } \\
\text { verse alterado por la presencia del } \\
\text { clínico }\end{array}$ & $\begin{array}{l}\text { Los animales pueden ser } \\
\text { entrenados para cooperar }\end{array}$ & Métodos invasivos \\
\hline Parámetros bioquímicos & $\begin{array}{l}\text { Pueden haber técnicas invasivas y } \\
\text { verse alterado por la presencia del } \\
\text { clínico }\end{array}$ & $\begin{array}{l}\text { Los animales pueden ser } \\
\text { entrenados para cooperar }\end{array}$ & Métodos invasivos \\
\hline
\end{tabular}

Fuente: [6]

Según la tabla anterior, existen diferentes maneras de evaluar el bienestar animal; sin embargo, en Colombia, no hay estudios concretos que evalúen el bienestar de estas especies. Además, no existen en el país parámetros establecidos que mejoren o establezcan condiciones de bienestar para estas especies. El sistema extensivo, aunque tiene algunas condiciones medioambientales favorables, no garantiza el bienestar animal, por lo que es probable que la producción en Colombia de ovejas y cabras no tenga en cuenta el bienestar animal.

Un estudio Delphi en el 2011 concluyó que los mayores porcentajes de problemas de bienestar y patologías en ovinos en las diferentes edades productivas son los siguientes: corderos muy jóvenes (castraciones, corte de cola laminitis y artritis séptica), corderos en crecimiento (parasitismo gastrointestinal, laminitis, miasis), ovejas (pobre condición corporal, problemas de nutrición, laminitis, distocia) y reproductores (pobre condición corporal, problemas de nutrición, laminitis) [8].

Un problema bien acentuado de bienestar animal en estas especies es el sacrificio. En Colombia, no existe normatividad para el sacrificio para los ovinoscaprinos, por lo que las plantas para bovinos se adaptan para los ovinos-caprinos. De igual manera, puede haber fallas en transporte, corrales, descanso, manejo en general, aturdimiento, sangría, entre otros, presentando posiblemente problemas de estrés al sacrificio y disminución en la calidad de la carne. Existen otras dificultades y vacíos relacionados con el manejo de ovinos, debilidad en las instalaciones, sacrificio de traspatio, condiciones sanitarias desfavorables, problemas sanitarios, la no existencia de programas de prevención y control de enfermedades, y sobre todo, la extrapolación del sistema productivo de los bovinos hacia los caprinos y ovinos, cuando cada una debería ser diferente y estar estandarizada.

\section{Factores que afectan la producción}

Son muchos los factores que afectan esta especie, por ejemplo:

- Bajo consumo de cárnicos, leche y productos derivados de estas especies.

- La no existencia de normatividad.

- Los intereses políticos para incrementar esfuerzos en la exportación de productos de las especies tradicionales (aves, bovinos, porcinos).

- El fortalecimiento de otros sectores pecuarios (mascotas, equinos, entre otros).

\section{Análisis de posibles cambios que podrían} mejorar la producción de esta especie

Algunos de los puntos para mejorar podrían ser:

- Creación de normatividad para todos los eslabones de la cadena productiva.

- Fortalecimiento y cohesión del sector, asociaciones, cooperativas de productores, créditos o proyectos para fomentar la industria ovino-caprina (proyectos productivos).

- Campañas para fomentar el consumo de cárnicos y derivados de esta especie, divulgando sus beneficios y la relación costo-beneficio.

- Acceso a financiación de proyectos para el diseño y construcción de plantas de beneficio diseñadas para estas especies.

- Mercadeo, implementación de estrategias de comercialización, acceso a mercados.

- Investigación básica y aplicada.

- Industrialización y tecnología en los sistemas productivos.

- Valor agregado a los productos. 
- Según lo mencionado por la FAO, los ovinos-caprinos (especies menores) son una adecuada estrategia para mitigar la pobreza y fortalecer la agricultura familiar.

- Formación de profesionales dedicados a la producción especializada de estas especies.

La demanda en el consumo podría favorecer la mejora de los sistemas de bienestar, el nacimiento de nuevas "marcas" comerciales que produzcan carne de ovinos-caprinos, la producción orgánica de alta calidad y el monitoreo del bienestar animal en las granjas productoras [8]. El monitoreo del bienestar en estas granjas o centros de producción (de ovinos y caprinos) se puede implementar, entre otras estrategias, mediante la verificación de indicadores, tales como: piel y pelo (lesiones, brillo), la condición corporal, la recogida para el ordeño, defecación y micción durante el ordeño, presencia de laminitis (ausencia de enfermedades en general), cojeras, comportamientos anormales, densidad de la población, prácticas de higiene en el ordeño y manejo, calidad del aire, temperatura y humedad relativas. De la misma forma, como hay indicadores que se pueden medir en la granja, existen otros que requieren métodos de laboratorio y otros que se deben medir en el sacrificio. Sin embargo, los indicadores que se tomen en la granja deben ser evaluados cuidadosamente, ya que la mayoría de estudios los reportan en granjas o condiciones experimentales [9-11].

Finalmente, tal como lo propone la European Welfare Quality Project, los indicadores de bienestar animal se pueden resumir en cuatro aspectos básicos: alojamiento adecuado, nutrición, salud y comportamiento normal o adecuado; son las condiciones mínimas que deben considerar las explotaciones de ovinos y caprinos, orientadas a producir una carne de calidad [12]. Estas condiciones dependen de la interacción entre el médico veterinario y los productores, los cuales deben trabajar en equipo para poder adaptarse a las condiciones socioeconómicas de los sistemas productivos, a las limitaciones económicas y a las condiciones de manejo existentes en el medio.

\section{Conclusiones}

El bienestar animal en Colombia presenta deficiencias en cuanto a su aplicación en los sistemas productivos, por lo que se deben incluir en la cadena ovino-caprina indicadores de bienestar animal.
Es necesario el trabajo y la asesoría del médico veterinario en la planificación de explotaciones ovinocaprinas para establecer desde el inicio condiciones adecuadas de bienestar animal en la explotación, mejorando la calidad del producto final (carne, leche, pieles, lana, crías) y la calidad de vida de los animales.

\section{Referencias}

[1] Espinal CF, Covaleda HM, Amezquita JE. La cadena de ovinos y caprinos en Colombia. Documento de trabajo n. ${ }^{\circ} 125$. Bogotá: Observatorio Agrocadenas Colombia. 2006.

[2] Albarracín WH, Sánchez, IB. Caracterización del sacrificio de corderos de pelo a partir de cruces con razas criollas colombianas. Rev mvz Córdoba. 2013;18(1):3370-8.

[3] Romero FA, Duarte JH. Identificación y evaluación nutricional de especies vegetales consumidas habitualmente por ovinos y caprinos en pastoreo en el desierto de la Tatacoa, Huila, Colombia. Agroforestería Neotropical. 2012;(2):4-18.

[4] Universidad Nacional de Colombia. Sistemas de producción de pequeños rumiantes. [Curso virtual] Disponible en: http://www.virtual.unal.edu.co/cursos/veterinaria/2017107_2/und_1/index.html

[5] Arévalo A., Correa G. Tecnología de la ovinocultura colombiana: estado del arte. Revista Ciencia Animal. 2013;6:125-142.

[6] Asociación de Criadores de Ganado Ovino de Colombia (Asoovinos). Plan estratégico para el desarrollo gremial 2010-2018. Plan estratégico de la ovinocultura colombiana 2018. Bogotá: Asoovinos; 2010. Disponible en: www.asoovinos.org

[7] Roger PA. The Impact of Disease and Disease Prevention on Sheep Welfare. Small Ruminant Research. 2008;76:104-11.

[8] Phythian CJ, Michalopoulou E, Jones PH, Winter AC, Clarkson MJ, Stubbings LA, et al. Validating Indicators of Sheep Welfare through a Consensus of Expert Opinion. Animal. 2011;5(6):943-52.

[9] Battini M, Peric T, Ajuda I, Vieira A, Grosso L, Barbieri S, et al. Hair Coat Condition: A Valid and Reliable Indicator for On-Farm Welfare Assessment in Adult Dairy Goats. Small Rum Res. 2015;123(2-3):197-203. 
[10] Caroprese M, Casamassima D, Rassu SP, Napolino F, Sevi A. Monitoring the On-Farm Welfare of Sheep and Goats. Ital J Anim Sci. 2009;8(1):343-54.

[11] Battini M, Vieira A, Barbieri S, Ajuda L, Stilwell G, Mattiello S. Invited Review: Animal-Based
Indicators for On-Farm Welfare Assessment for Dairy Goats. J Dairy Sci. 2014;97(11):6625-48.

[12] Stafford K. Sheep Veterinarians and the Welfare of Sheep: No Simple Matter. Small Rum Res. 2014;118:106-9. 